\title{
LAS JUVENTUDES DE SECTORES MEDIOS DE TIERRA DEL FUEGO: EXPLORANDO LOS EFECTOS DEL COVID-19 Y EL AISLAMIENTO DESDE UNA ESCALA SUBNACIONAL
}

\author{
BRUNO COLOMBARI ${ }^{1}$ \\ LÍBERA GUZZI ${ }^{2}$ \\ LUCILA KIDA ${ }^{3}$
}

\begin{abstract}
RESUMEN
En este artículo nos preguntamos por los efectos generados por el brote de Covid-19 y el confinamiento exigido en jóvenes de sectores medios de la Provincia de Tierra del Fuego, Argentina. Desde una metodología cuantitativa, analizamos datos primarios construidos mediante una encuesta en línea aplicada en septiembre de 2020 a personas de 18 a 29 años. A partir de allí, identificamos una serie de consecuencias educativas, laborales, de salud y de uso del tiempo que, en algunos casos, dependen de las particularidades provinciales. Concluimos que la crisis por la pandemia incide en las posibilidades de emancipación familiar y proyección personal.
\end{abstract}

PALABRAS CLAVES: JUVENTUDES, PANDEMIA, TIERRA DEL FUEGO.

\footnotetext{
${ }^{1}$ Maestrando en Desarrollo Territorial y Urbano de la Universidad Nacional de Quilmes, Argentina. Diplomado en Estudios y Políticas de Juventud (FLACSO), Licenciado y Profesor en Sociología de la Universidad de Buenos Aires (UBA). DocenteInvestigador de la Universidad Nacional de Tierra del Fuego, Argentina (UNTDF); Coordinador de la Licenciatura en Sociología y Director del proyecto «Condiciones juveniles insulares. Un estudio sobre las formas de sociabilidad de jóvenes en la Provincia de Tierra del Fuego, Antártida e Islas del Atlántico Sur» (UNTDF). Correo electrónico: bcolombari@untdf.edu.ar

2 Doctoranda en Ciencias Sociales de la UBA, Magíster en Comunicación y Cultura Contemporánea por la Universidad Nacional de Córdoba y Licenciada en Comunicación Social. Docente-Investigadora de la UNTDF e integrante del proyecto mencionado. Correo electrónico: lguzzi@untdf.edu.ar

${ }^{3}$ Especialista en Políticas Educativas por la Universidad Torcuato Di Tella y Licenciada en Ciencia Política (USAL). Docente-Investigadora de la UNTDF, integrante del proyecto mencionado. Correo electrónico:mlkida@untdf.edu.ar
} 


\title{
AS JUVENTUDES DE CLASSE MÉDIA DE TERRA DO FOGO: EXPLORANDO OS EFEITOS DA COVID-19 E O ISOLAMENTO A PARTIR DE UMA ESCALA SUBNACIONAL
}

\begin{abstract}
RESUMO
Neste artigo nos perguntamos sobre os efeitos gerados pelo surto de COVID-19 e o confinamento exigido nos jovens de classe média da Província de Terra do Fogo, Argentina. A partir de uma metodologia quantitativa, analisamos dados primários construídos através de uma pesquisa online aplicada em setembro de 2020 a pessoas com idades compreendidas entre os 18 e os 29 anos. Logo disso, identificamos uma série de consequências em termos de educação, emprego, saúde e o uso do tempo que, em alguns casos, dependem das particularidades provinciais. Concluímos que a crise provocada pela pandemia incide nas possibilidades de emancipação familiar e de projeção pessoal.

PALAVRAS-CHAVE: JUVENTUdE, PANDEMIA, TERRA DO FogO.

\section{MIDDle-ClASS YOUTHS OF TIERRA DEL FUEGO: EXPLORING THE EFFECTS OF COVID-19 AND ISOLATION FROM A SUBNATIONAL SCALE}

\begin{abstract}
In this article, we inquire into the effects generated by the outbreak of COVID-19 and the obligatory lockdown for middle-class young people from Tierra del Fuego, Argentina. From a quantitative methodology, we analyzed primary data collected from an online survey conducted to people aged 18-29 years old in September 2020. We identified a series of education, work, health, and time-related consequences, some of them depending on provincial peculiarities. We conclude that the crisis caused by the pandemic has an impact on the possibilities of family emancipation and personal future plans.
\end{abstract}

KEYWORDS: YOUTHS, PANDEMIC, TIERRA DEL FUEGO. 


\section{INTRODUCCIÓN}

El brote de Covid-19 en diciembre de 2019 y su rápida expansión mundial desencadenó una serie de rigurosos procedimientos multiescalares de restricción a la circulación de personas (para ralentizar la diseminación del virus), que generaron efectos negativos sobre diversas actividades económicas, educativas y también en la salud física y mental de las personas ${ }^{4}$. Las medidas de aislamiento social establecidas por los gobiernos en respuesta a la situación implicaron cambios drásticos en los hábitos de vida. Por otra parte, las condiciones para afrontar la crisis desatada por la pandemia son muy dispares y dependen principalmente de factores económicos, sanitarios y etarios, entre otros. En relación con el último factor, cabe señalar que para las personas jóvenes, el impacto del Covid-19 tiene una menor letalidad que para el resto de la población (Ferguson et al. en OIT, 2020), ya que una vez contraído el virus, la probabilidad de muerte es superior en el caso de las personas mayores (Baqui et al. en CEPAL, 2020). Sin embargo, a pesar de que no son los más afectados por la enfermedad, la situación epidemiológica produjo profundas consecuencias en otros aspectos de la vida, ya que para muchas/os generó la interrupción de procesos educativos y de formación (debido al cierre masivo de las actividades presenciales de instituciones educativas) e incrementó su vulnerabilidad laboral producto del deterioro económico y del mercado laboral, con su consecuente impacto en las posibilidades de emancipación familiar y en las proyecciones de movilidad social $^{5}$.

Este panorama surge de las investigaciones desarrolladas, principalmente desde organismos internacionales (como la Organización Internacional del

\footnotetext{
${ }^{4}$ El 11 de marzo de 2020 la Organización Mundial de la Salud (OMS) declaró como pandemia el brote de Covid-19.

${ }^{5}$ Según datos de la Organización de las Naciones Unidas para la Educación, la Ciencia y la Cultura (UNESCO 2020), a mediados de mayo de 2020 más de 160 millones de estudiantes de todos los niveles de enseñanza, en América Latina y el Caribe, habían dejado de tener clases presenciales en la escuela.
} 
Trabajo, la Organización de las Naciones Unidas para la Educación, la Ciencia y la Cultura, y la Comisión Económica para América Latina y el Caribe), que giran en torno al análisis de las consecuencias laborales, educativas y de salud mental en términos globales, nacionales y regionales, siendo minoritarios los estudios que construyeron registros de los efectos a escala subnacional ${ }^{6}$. Asimismo, la pandemia y las medidas de aislamiento social obstaculizaron la producción de datos sobre los efectos experimentados, particularmente, en las generaciones jóvenes y a escala local o provincial. Ante la escasa información sobre las experiencias de este sector de la población en este contexto, los registros de las percepciones y experiencias juveniles son un aporte para identificar y visibilizar las dificultades y vulnerabilidades que permitan orientar las acciones gubernamentales ${ }^{7}$. Sabemos que las formas de ser joven no son iguales en los distintos países, pero tampoco en las distintas regiones, ciudades y segmentos socioeconómicos. En este sentido, los registros realizados que den cuenta de las consecuencias de la crisis a partir de las particularidades territoriales tienen un importante valor al permitir observar, por un lado, los efectos similares más allá de los contextos específicos $\mathrm{y}$, por otro, las consecuencias que responden precisamente a las dinámicas particulares del entorno.

Con el propósito de proveer de base empírica al fenómeno descrito, el presente estudio cuantitativo descriptivo tiene por objetivo analizar, desde una

${ }^{6}$ Entre estas investigaciones cabe destacar los informes: a) «Los jóvenes y la covid-19: efectos en los empleos, la educación, los derechos y el bienestar mental», desarrollado por la OIT y en el que participaron jóvenes de 112 países, aunque los datos expresados no están desagregados por país sino por «total mundial»; b) «La educación en tiempos de la pandemia de covid-19», en el que se relevó la situación en treinta y tres países de América Latina y el Caribe donde los datos están desagregados a nivel nacional.

${ }^{7}$ Cabe señalar el planteo realizado por Martín Criado (2009) sobre la forma en que las encuestas han operado desde el campo estatal y desde las ciencias sociales en tanto instrumento para la «imposición de problemáticas» sobre la juventud. El autor señala que quienes preguntan y relevan imponen los temas, condicionan las respuestas y se erigen en los traductores de lo que las/os jóvenes piensan y quieren. Más allá de las alertas identificadas por Criado, las encuestas permiten relevar y recopilar información sobre una multiplicidad de temáticas. 
perspectiva multidimensional y en una escala subnacional, los efectos generados durante los primeros meses de la pandemia por Covid-19 en las/os jóvenes, tomando como objeto de estudio las percepciones y experiencias de personas de 18 a 29 años de edad residentes en la provincia de Tierra del Fuego, Antártida e Islas del Atlántico Sur, Argentina. Mediante los datos producidos a través de una encuesta en línea autoadministrada, aplicada en septiembre de 2020, se describe el impacto de la pandemia en jóvenes, sobre la base de cuatro dimensiones analíticas: trabajo e ingresos; educación y conectividad; salud; ocio y uso del tiempo libre. En este sentido, el análisis presentado describe, mediante una selección limitada sobre una multiplicidad de hechos sociales que podrían constituirse como problemáticos, la manera en que la pandemia afectó (y continúa afectando) la vida de un sector de la población joven que reside en un territorio insular, periférico y de lejanía de los principales centros urbanos ${ }^{8}$.

El artículo se encuentra estructurado de la siguiente manera. En primer lugar, se presentan algunos estudios referidos a la población joven residente en la provincia. Luego, se detallan las especificaciones técnicas del relevamiento cuantitativo y se caracterizan las unidades muestrales con el propósito de delimitar el alcance de los resultados. En tercer lugar, sobre la base de esos resultados, se describen las percepciones y experiencias de las/os respondientes para dar cuenta de los efectos que la pandemia y el aislamiento generaron, de acuerdo con las cuatro dimensiones mencionadas. Finalmente, a partir del análisis desarrollado, se presentan algunas conclusiones orientadas a articular los resultados obtenidos en las cuatro dimensiones analizadas e identificar las particularidades locales de la crisis en jóvenes de sectores medios.

\footnotetext{
${ }^{8}$ La provincia se encuentra enclavada en la Isla Grande de Tierra del Fuego, y su lejanía de los grandes centros urbanos, sumada a la necesidad de atravesar la frontera y el Estrecho de Magallanes para su conexión terrestre con el continente, agregan a la población que la habita un doble aislamiento: el geográfico, basado en su característica de ultramar, y el dispuesto con el objetivo de garantizar el cuidado de la salud (Colombari, Hermida y Guzzi, 2020).
} 


\section{ESTUdios DE JUVENTUdES EN TIERRA DEL FUEGO}

Una de las tesituras que más ha ayudado a ampliar y complejizar el campo de estudios sobre juventudes es aquella según la cual no hay una juventud. Esto implica, entre otras cosas, asumir el hecho de que dicho término cobra múltiples sentidos según el contexto histórico, social, económico, político y cultural en que las/os jóvenes viven. Con ello se apunta a abandonar aquel concepto vacío de contexto, neutral y estático, vinculado casi exclusivamente a cuestiones biológicas, que tiende a unificar su heterogeneidad social (Krauskopf, 2004). De este modo, el período juvenil tendrá distintos sentidos según cada sociedad, en función de los distintos sectores socioeconómicos y la diversidad de manifestaciones culturales. Consideramos a la noción de juventud en tanto «experiencia vital y categoría socio-histórica definida en clave relacional, más que etaria o biológica» (Vommaro, 2014: 16). El proceso de construcción de identidad de cada individuo se configura de este modo como uno de los elementos característicos y nucleares del período juvenil, proceso que se asocia a condicionantes individuales, familiares, sociales, culturales e históricos determinados. Es un proceso complejo que se constata en diversos niveles y de manera simultánea.

Cabe destacar que los estudios de juventud en Tierra del Fuego cobran importancia al ser esta una de las provincias con mayor población joven (15-29 años) del país, y la segunda después de Santa Cruz en la región Patagónica. Asimismo, para el censo 2010 el porcentaje de jóvenes residentes se encontraba por encima de la media nacional $(26 \% \text { y } 25 \% \text {, respectivamente })^{9}$. Esta significativa presencia de personas jóvenes residentes en la provincia, sumada a las características específicas de los modos de habitar en ella - el hecho de que se trate de una región insular y con bajas temperaturas; «aislada» respecto a los

\footnotetext{
${ }^{9}$ Se utilizan datos del censo de población 2010, ya que el previsto para 2020 fue
} suspendido por la situación epidemiológica producto de la pandemia por Covid-19. 
principales centros de poder político y económico; construida como un territorio de fronteras físicas y políticas; con un desarrollo de la industria electrónica a través de la Ley de Promoción Industrial; con altos ingresos de una parte de sus habitantes, entre otros- intervienen en la configuración de los modos posibles de habitar el espacio social. Cabe agregar que en investigaciones previas (Colombari et al., 2020) se identificó que la mayoría de la población joven en Tierra del Fuego nació fuera de esta, lo que le imprimió un rasgo característico a las políticas de juventud locales: la promoción de la permanencia y el arraigo de las personas jóvenes en el territorio insular mediante diversas políticas públicas.

En lo que respecta al mercado laboral, es sabido que la población joven presenta una mayor vulnerabilidad a las fluctuaciones de la economía que el resto de la población económicamente activa (Jacinto, 2010). Al analizar la situación local, en los momentos de retracción y de caída de la demanda de mano de obra, Colombari, Hermida y Picón (2019) indican que la tasa de desempleo de la población joven crece con mayor intensidad, triplicando la tasa de desempleo general. Los mismos autores señalan que, a lo largo del período 2003-2018, se dio un crecimiento de la participación en la población ocupada de aquellas/os jóvenes que poseen mayores credenciales educativas ${ }^{10}$. En el desarrollo de este trabajo daremos cuenta de los efectos de la pandemia en el contexto local, teniendo en cuenta estas particularidades.

\footnotetext{
${ }^{10}$ Las/os jóvenes con «secundario completo y superior incompleto» crecen en su participación dentro de la población ocupada de un 58,7\% en 2003 a un $68,8 \%$ en 2018, mientras que las/os jóvenes con «hasta secundario incompleto» caen de un $40,4 \%$ en 2003 a un $20,6 \%$ en 2018. En relación con los que cuentan con nivel «superior completo» puede observarse un importante crecimiento de su participación en la población ocupada, pasando de un 1\% en 2003 a un 10,5\% en 2018 (Colombari, Picón y Hermida, 2019).
} 


\section{Metodología deL RELEVAMiento Y}

\section{CARACTERÍSTICAS DE LA MUESTRA}

El abordaje metodológico se basó en fuentes de datos mixtas. Si bien se recurrió a datos secundarios producidos desde diversos organismos nacionales e internacionales, la descripción se realizó sobre la base de los resultados de una encuesta en línea autoadministrada, aplicada entre el 4 y el 29 de septiembre de 2020 que respondieron 363 personas, reclutadas mediante las redes interpersonales del equipo de investigación que compusieron una muestra no probabilística $^{11}$. La población comprendió a jóvenes de 18 a 29 años de edad de las principales localidades de la provincia (Ushuaia, Río Grande y Tolhuin) ${ }^{12}$.

Más allá de los impedimentos de aplicación de una encuesta presencial, la encuesta en línea es una alternativa accesible debido a la relativa facilidad de su programación, sus bajos costos, la optimización de tiempos de realización y su mayor amplitud geográfica. Asimismo, tal como señalan Melegarie y Fernández (2019), responde con buen rendimiento en aquellos espacios en los que se comunican e interactúan las generaciones más jóvenes (e-mail y redes sociales).

En relación con las características sociodemográficas de la muestra, las mujeres representan el 49\% mientras que los varones tienen una representación ligeramente mayor $(51 \%)^{13}$. En relación con los subgrupos de edad, las/os

${ }^{11}$ El reclutamiento se realizó mediante Whatsapp y redes sociales (Facebook, Instagram y Twitter) del equipo de investigación.

${ }^{12}$ Si bien la encuesta estuvo orientada a jóvenes de 15 a 29 años (rango estipulado por la Dirección Nacional de Juventud para definir a las personas jóvenes), la participación de respondientes de 15 a 17 años fue casi nula, por lo que decidió excluirse dicho rango etario del análisis realizado.

${ }^{13}$ Según datos del Censo Nacional de Población, Hogares y Viviendas 2010, las personas de entre 18 y 29 años de edad totalizan 26.172 , lo que representa el $20,5 \%$ de la población total (127.205). Los varones de este rango etario totalizan $13.571(52 \%) \mathrm{y}$ las mujeres, 12.601 (48\%). 
jóvenes de 18 a 21 años representan el 40\%, las/os de 22 a 25 el 31\% y las/os de 26 a 29 el 29\%. La mayor parte de las/os respondientes convive con su padre/madre (59\%), seguido de quienes lo hacen con su pareja (23\%). El porcentaje restante presenta otras formas de convivencia (amigas/os y otros familiares). Con respecto a las características socioeconómicas, la muestra estuvo compuesta mayoritariamente por jóvenes: a) laboralmente activos (73\%), de las/os cuales un 54\% estaba ocupado y un 19\% desocupado; b) que cuentan con secundario completo $(40 \%)$ y universitario incompleto $(37 \%)^{14}$; c) que disponen de los dispositivos tecnológicos adecuados para responder una encuesta de estas características, ya que un $86 \%$ cuenta con $W i-F i$ y computadora; y d) que habitan viviendas que no presentan situaciones de hacinamiento crítico $(98,5 \%)$, es decir, aquellas en las cuales más de tres personas comparten una misma habitación. En la siguiente tabla se presenta de manera esquemática la información desarrollada.

CUADRO 1. CARACTERÍSTICAS GENERALES DE LAS/OS JÓVENES RESPONDIENTES

\begin{tabular}{|c|c|c|c|}
\hline \multicolumn{2}{|c|}{ Condición de actividad } & \multicolumn{2}{|l|}{ Disponibilidad de PC y conectividad } \\
\hline Activos & $73 \%$ & $\begin{array}{l}\text { Dispone de computadora y conexión } \\
W i-F i\end{array}$ & $86 \%$ \\
\hline Inactivos & $27 \%$ & $\begin{array}{l}\text { No dispone de computadora y conexión } \\
W i-F i\end{array}$ & $14 \%$ \\
\hline Total & $\begin{array}{c}100 \\
\%\end{array}$ & $\begin{array}{ll} & \text { Total }\end{array}$ & $100 \%$ \\
\hline \multicolumn{2}{|l|}{ Nivel educativo } & \multicolumn{2}{|l|}{$\begin{array}{r}\text { Situación habitacional } \\
\end{array}$} \\
\hline Secundario incompleto & $9 \%$ & Sin hacinamiento crítico & $\begin{array}{c}98,5 \\
\%\end{array}$ \\
\hline Secundario completo & $40 \%$ & Con hacinamiento crítico & $1,5 \%$ \\
\hline Universitario incompleto & $37 \%$ & Total & $100 \%$ \\
\hline Universitario completo & $14 \%$ & & \\
\hline Total & $\begin{array}{c}100 \\
\%\end{array}$ & & \\
\hline
\end{tabular}

Fuente: Elaboración propia, sobre la base de resultados de la encuesta.

\footnotetext{
${ }^{14}$ Una porción minoritaria cuenta con nivel universitario completo (14\%) y secundario incompleto $(9 \%)$.
} 
A partir de estos datos, puede concluirse que tres cuartas partes de la muestra comparten características relativamente homogéneas en términos laborales, educativos, de accesibilidad a dispositivos tecnológicos y de comunicación, y de habitabilidad, lo que permite inducir que, en términos socioeconómicos, se trata de respondientes de sectores medios.

\section{PRESentación De los ReSUltados: los EFECTOS \\ LOCALES DE LA PANDEMIA EN LAS/OS JÓVENES DE \\ SECTORES MEDIOS RESIDENTES EN TIERRA DEL FUEGO, Argentina}

La encuesta permitió abordar los efectos de la pandemia en las/os jóvenes de la provincia mediante cuatro dimensiones analíticas: trabajo e ingresos; educación y conectividad; salud; ocio y uso del tiempo libre. Esta multidimensionalidad es puesta en juego mediante el entrecruzamiento de diversos aspectos de cada dimensión, con el propósito de complementar las lecturas realizadas. Asimismo, los datos primarios se relacionaron con registros secundarios para abordar con mayor detalle los efectos particulares de la pandemia a partir del contexto provincial y también los efectos similares registrados por estudios de escala nacional e internacional.

\subsection{TRABAJO E INGRESOS}

Una de las dimensiones relevadas en la encuesta está vinculada al trabajo y los ingresos de las/os jóvenes. Todos los aspectos indagados — condición de actividad, categoría ocupacional, tipo de establecimiento, beneficios laborales, percepción de asistencias estatales, ingresos laborales, cantidad de horas trabajadas - estuvieron orientados a dar cuenta del impacto de la pandemia, 
poniendo especial foco en el modo en que la población de sexo femenino se vio afectada, en tanto constituye un sector especialmente vulnerable en lo que refiere al mundo del trabajo.

Del $54 \%$ de jóvenes activas/os que se encontraban ocupadas/os al momento de la encuesta, un $76 \%$ trabajaba como empleada/o u obrera/o para un patrón, empresa o institución (entre ello/as, 60\% eran varones y 40\% mujeres); un $15 \%$ era cuentapropista (48\% varones y 52\% mujeres); un $6 \%$ era trabajadora/or familiar (38\% varones y $62 \%$ mujeres), y un 3\% era trabajadora/o del servicio doméstico (mujeres en su totalidad). Además, una mayoría trabajaba en el sector privado (58\%) y contaba con beneficios laborales (57\% en total, de las/cuales, $59 \%$ eran varones y $41 \%$ mujeres $)^{15}$. Estos datos ofrecen una primera aproximación a las diferencias y desigualdades que existen entre varones y mujeres, y que estructuran en términos generales el mercado de trabajo. Por otro lado, excluyendo a las personas inactivas, la desocupación alcanzaba a un cuarto de las/os jóvenes respondientes, y afectaba en mayor medida a las mujeres que a los varones, en tanto la tasa de desocupación llegaba a un 30\% en las personas de sexo femenino, contra un $20 \%$ en las de sexo masculino.

La situación reflejada en lo que refiere a las tasas de ocupación y desocupación guarda relación con el panorama del mercado laboral del contexto prepandemia y muestra una cierta profundización de los problemas vinculados al trabajo para la población joven: según la información de la Encuesta Permanente de Hogares (EPH) del Instituto Nacional de Estadísticas y Censos (INDEC) ${ }^{16}$, al

${ }^{15}$ Tales como aguinaldo, obra social, aportes, seguro, etc., generalmente asociados al trabajo registrado.

${ }^{16} \mathrm{La}$ EPH es un estudio que se desarrolla en conjunto entre el INDEC y los organismos de estadísticas de cada provincia del país, orientado a la producción de indicadores sociodemográficos y socioeconómicos de la población. Se trata de una encuesta por muestreo, que se realiza en todo el país y se aplica cuatro veces al año. 
tercer trimestre de $2019^{17}$, la tasa de desocupación en nuestra provincia era del $22,8 \%$ para los varones de 14 a 29 años y del $25,6 \%$ para las mujeres de esa edad (INDEC, 2019). Sin embargo, al profundizar en el análisis de los datos producidos por nuestra encuesta — especialmente aquellos vinculados con la cuestión de los ingresos - se observa que el impacto de la pandemia no se circunscribe a los problemas de acceso y sostenimiento del trabajo, sino que los efectos de la crisis han alcanzado significativamente también a quienes se encontraban ocupados en ese momento.

En primer lugar, resulta útil referirnos a la asistencia estatal durante el período de aislamiento, ya que más de un tercio de las/os respondientes de la encuesta recibió alguna asistencia (36\%). Dentro de este grupo, un 22\% indicó que percibió al menos una, el 10\% dos y el 3\% tres tipos de asistencias diferentes. La más percibida fue la asistencia alimentaria (30\%), provista por los gobiernos locales (municipales y provincial) ${ }^{18}$; y la segunda el Ingreso Familiar de Emergencia (IFE), un tipo de asignación estatal extraordinaria ofrecida por el gobierno nacional en el marco de la situación de pandemia ${ }^{19}$. En menor proporción, las/os jóvenes percibieron transferencias monetarias o asignaciones

${ }^{17}$ Hemos tomado este período para ilustrar el contexto prepandemia ya que, teniendo en cuenta el momento en el que se realizó el relevamiento (septiembre de 2020), es posible analizar la variación interanual, destacando el impacto de la pandemia y el aislamiento, más allá de otros factores de carácter estacional. Para el tercer trimestre de 2020 no hay información disponible debido a problemas técnicos en el desarrollo de la EPH.

${ }^{18}$ Este tipo de asistencia apunta a asistir o complementar la alimentación de familias en situación de vulnerabilidad social y/o inseguridad alimentaria, a través de la entrega de módulos alimentarios (productos de consumo habitual de diverso tipo), tarjeta para la compra de alimentos, etc.

${ }^{19}$ El IFE fue una prestación monetaria de carácter excepcional destinada a compensar la pérdida o grave disminución de ingresos de personas afectadas por la situación de emergencia sanitaria por Covid-19, como trabajadores autónomos, de casas particulares e informales. Consistió en el cobro de tres asignaciones mensuales de diez mil pesos argentinos cada una (aproximadamente unos 100 dólares) para personas entre 18 a 65 años que no percibieran pensiones ni subsidios y que no tuvieran otro ingreso. 
existentes previamente. A modo de hipótesis puede pensarse que la pandemia generó que sectores que habitualmente no recurren a ayudas estatales hayan debido solicitarlas, en la mayoría de los casos con el fin de atender necesidades de primer orden como la alimentación, debido a la situación de vulnerabilidad e incertidumbre planteada por el contexto.

En segundo lugar, consultadas/os acerca de sus ingresos, si bien la mayoría manifestó que no tuvieron modificaciones, un $31 \%$ indicó que se redujeron. Este impacto negativo afectó mayoritariamente a las mujeres, entre las cuales la merma llegó al 40\%. La disminución de ingresos en un tercio de las/os encuestados da cuenta del alcance de la crisis económica, en tanto incluso muchas/os de quienes mantuvieron el trabajo durante la pandemia se enfrentaron a esta reducción, con su consecuente impacto en el poder adquisitivo y en la economía familiar. En tercer lugar, al indagar acerca de los motivos por los cuales no trabajaron durante el aislamiento, un 16\% indicó que adquirió la condición de desocupado/a durante la pandemia, ya sea porque perdió el trabajo $(9,5 \%)$ o bien porque no pudo desarrollar su actividad por cuenta propia de forma habitual como consecuencia del contexto $(6,5 \%)$.

\section{CUADRO 2. EFECTOS DE LA PANDEMIA EN EL TRABAJO Y EN LOS INGRESOS DE} LAS/OS RESPONDIENTES

\begin{tabular}{|c|c|c|}
\hline Ingresos & Mujeres & Varones \\
\hline Mis ingresos aumentaron & $10 \%$ & $14 \%$ \\
\hline Mis ingresos se mantuvieron igual & $49 \%$ & $59 \%$ \\
\hline Mis ingresos se redujeron & $40 \%$ & $27 \%$ \\
\hline Total & $100 \%$ & $100 \%$ \\
\hline \multicolumn{3}{|c|}{ Motivos por los cuales no trabajó durante el aislamiento } \\
\hline \multicolumn{2}{|c|}{$\begin{array}{l}\text { He perdido mi trabajo o actividad laboral como consecuencia de } \\
\text { la pandemia }\end{array}$} & $9,5 \%$ \\
\hline \multicolumn{2}{|c|}{ No he logrado realizar mi actividad laboral de forma usual } & $6.5 \%$ \\
\hline \multicolumn{2}{|c|}{ No logro ni he logrado conseguir trabajo } & $84 \%$ \\
\hline & Total & $100 \%$ \\
\hline
\end{tabular}

Fuente: Elaboración propia, sobre la base de resultados de la encuesta. 
Finalmente, con relación a las/os jóvenes ocupados, un 29\% manifestó que trabajó menos horas durante el aislamiento, con un impacto ligeramente mayor sobre las mujeres (54\%) que sobre los varones (46\%). Del total de quienes se encontraron en esta situación, es decir, quienes trabajaron menos horas, un $60 \%$ indicó que las causas de esta reducción fueron principalmente restricciones establecidas por la empresa o institución en la que trabaja; en segundo lugar, falta de pedidos o clientes (21\%) y, en tercer lugar, la necesidad de cuidar de otras personas $(13,5 \%)$. Este último dato permite considerar la incidencia en la situación laboral de las/os jóvenes de factores no vinculados directamente con la economía, pero sí con las medidas de aislamiento. En efecto, consultadas/os acerca de la principal dificultad que enfrentaron las/os respondientes para desarrollar actividades durante este período, un 14\% indicó que la mayor dificultad fue el cuidado de niñas/os o adultas/os mayores, pero en el caso de las mujeres la proporción llega al 20\%. Esto confirma también que las tareas, actividades y servicios incluidos en las denominadas economías del cuidado (Rodríguez Enríquez, 2007), que recaen mayormente en las mujeres, se acrecentaron sustancialmente durante el aislamiento debido al drástico cambio en las rutinas, profundizando aún más las desigualdades de género que existen en el mundo del trabajo (Bidaseca et al., 2020).

A modo de síntesis, es posible señalar que la crisis por Covid-19 afectó significativamente a una parte de la población juvenil en términos de trabajo e ingresos, lo que incide en las posibilidades de autonomización familiar, y otros aspectos significativos de sus expectativas y proyecciones a futuro.

En relación con los ingresos, los datos específicos analizados conforme a otros datos de la encuesta dan cuenta de la incidencia en las trayectorias emancipatorias recorridas y proyectadas a mediano y largo plazo: por un lado, la mayor parte de las/os respondientes convivía con sus padres (59\%) al momento del relevamiento; por otro lado, al ser consultadas/os acerca de si debieron 
realizar movilidades en las viviendas que habitaban producto de la pandemia, un quinto $(21 \%)$ respondió afirmativamente, ya sea porque debió mudarse dentro de la provincia o de la misma ciudad (12\%), o bien porque debió regresar a Tierra del Fuego (9\%). Si bien la encuesta no indagó acerca de los motivos de estos movimientos, podemos suponer que estuvieron asociados a la decisión de transitar la pandemia y el aislamiento cerca de su familia y/o por motivos económicos.

En lo que refiere al trabajo, nuestro análisis, en primer lugar, confirma lo que diversos estudios sobre juventudes han mostrado y conceptualizado en cuanto a la mayor vulnerabilidad de las personas jóvenes — y dentro de ellas, más aún las mujeres - respecto al resto de la población económicamente activa. En segundo lugar, confirma las tendencias planteadas en un estudio previo acerca del mercado de trabajo joven en la provincia, en cuanto a que este sector precisamente por su vulnerabilidad - se ve afectado en mayor medida en los momentos de crisis y caída de la actividad económica, ya que «por su menor costo de despido y su rol periférico respecto de las actividades centrales, las y los jóvenes suelen ser la variable de ajuste» (Colombari et al., 2019: 19). En tercer lugar, en el caso de Tierra del Fuego, nuestro estudio abre un alerta en torno al impacto que la pandemia podría tener en un proceso estructural de precariedad y dificultad en el acceso al trabajo. Esto, teniendo en cuenta que la crisis sanitaria y económica asociada al Covid-19 ocurrió en un contexto previo de crisis y caída de la actividad — con alta inflación, pérdida generalizada del poder adquisitivo de los salarios y aumento del desempleo-, producto de las políticas implementadas por el gobierno del expresidente Macri y que, en nuestra provincia, había afectado especialmente a las/os jóvenes en virtud de una serie de medidas que impactaron negativamente en la industria electrónica local, radicada principalmente en la ciudad de Río Grande. Si a ello sumamos la paralización casi total de la actividad turística - muy importante en la ciudad de Ushuaia - a partir de la pandemia (Guzzi et al., 2020), lo que observamos es un 
panorama sumamente crítico.

\subsection{EDUCACIÓN Y CONECTIVIDAD}

En esta sección se indagan las consecuencias de la pandemia y las medidas de aislamiento sobre la educación de las/os jóvenes a partir de la encuesta llevada a cabo. También se exploran cuestiones relativas a la conectividad a internet, condición para la continuidad educativa en pandemia.

En primer lugar, respecto a la asistencia a establecimientos educativos, el $75 \%$ de las/os encuestadas/os continuó con sus actividades educativas formales durante el contexto de aislamiento (el 15\% no desarrollaba ninguna actividad educativa). Este alto nivel de permanencia en el sistema educativo coincide con los resultados de la Encuesta Nacional del Proceso de Continuidad Pedagógica (ENPCP), que indica que el $95 \%$ de los hogares encuestados recibieron propuestas de continuidad pedagógica ${ }^{20}$. A partir de aquí, dado que la mayoría $(62 \%)$ de quienes continuaron sus estudios durante la situación epidemiológica son estudiantes del nivel universitario, los datos que se presentan remiten únicamente a este grupo.

Un segundo aspecto que se indagó a través de la encuesta fueron las diversas formas en que las/os jóvenes que continuaron sus estudios durante la pandemia valoraron: a) los aprendizajes de contenidos curriculares; b) la comunicación con docentes y compañeras/os; y c) la organización del tiempo para realizar las tareas educativas. La escala de medición incluyó las categorías peor, igual o mejor que antes, con el propósito de comparar con la situación

\footnotetext{
${ }^{20}$ La ENPCP fue llevada a cabo en julio de 2020 por el Ministerio de Educación Nacional a través de una encuesta en línea, representativa a nivel nacional y jurisdiccional para caracterizar el proceso de continuidad pedagógica en el contexto de aislamiento en los niveles de enseñanza obligatorios.
} 
prepandemia. Los resultados muestran que en su mayoría las/os estudiantes evaluaron que sus aprendizajes y la forma de organizar el tiempo para realizar las tareas empeoraron, mientras que la comunicación con docentes y compañeras/os no se vio significativamente alterada.

Se destaca la valoración negativa que realizan de la situación educativa durante la pandemia. Trucco y Palma (2020) sostienen que la virtualidad conlleva numerosos cambios en los procesos de enseñanza y aprendizaje que afectan a las/os estudiantes, entre ellos la dificultad de sostener una relación pedagógica desde la virtualidad y la falta de preparación del profesorado para promover la continuidad de estudios en esta modalidad. A partir de la valoración realizada por las/os respondientes, puede sugerirse que la virtualización y el contexto de pandemia en general afectaron negativamente aspectos significativos del proceso de enseñanza-aprendizaje de una porción importante de jóvenes que continuaron sus estudios.

No obstante, la virtualización supuso al mismo tiempo la posibilidad de incorporar o mejorar el uso de herramientas digitales para actividades educativas, lo que contribuye al desarrollo de habilidades específicas que pueden ser utilizadas más allá del ámbito educativo. Con relación a esto, la encuesta realizada permite obtener información acerca de cómo las/os jóvenes encuestados valoraron el uso de plataformas educativas (EdModo, Classroom, Moodle) y de reuniones virtuales (Zoom, Google Meet); comparando el momento pre y durante la pandemia. La mayoría considera que el uso de estas herramientas mejoró, siendo mayor la frecuencia de quienes consideraron esta mejora respecto a reuniones virtuales $(59 \%)$ que quienes lo hicieron respecto a plataformas educativas $(55 \%)$.

Cabe destacar que una proporción no menor de jóvenes comenzaron a utilizar durante la pandemia plataformas educativas (14\%) y plataformas para reuniones virtuales (casi 19\%). Los datos indican que quienes empleaban estas 
herramientas con anterioridad a la pandemia intensificaron su uso, y quienes no lo hacían comenzaron a mediar sus experiencias educativas con estas. Aquí, cabe preguntarse hasta qué punto la virtualización, forzada por las circunstancias sanitarias, ha disminuido la brecha de acceso y dominio de herramientas digitales $^{21}$.

Otro aspecto relevante para analizar es el acompañamiento brindado por diferentes actores educativos en el contexto del aislamiento. Los organismos internacionales (CEPAL, 2020; UNESCO, 2018) indican que en situaciones de emergencia, las escuelas y el personal docente son actores fundamentales para el apoyo de las/os estudiantes, no solamente en términos pedagógicos, sino también emocionales. Las/os docentes, en su caso, procuran abordar estos retos desde recursos desiguales, como lo son la conectividad a internet y la disponibilidad de dispositivos electrónicos (UNESCO, 2018; ENPCP, 2020); pese a lo cual la encuesta estaría mostrando que cumplen una función social importante. En el caso estudiado, una mayoría de estudiantes valoró de manera positiva el acompañamiento recibido por parte del establecimiento educativo (61\%), de las/os docentes (75\%), de las/os compañeros (61\%) y de los miembros del hogar $(85 \%)$

A modo de cierre, puede señalarse que a pesar del alto nivel de continuidad de los estudios durante el contexto de pandemia, ciertos aprendizajes $\mathrm{y}$ prácticas fueron percibidos como obstaculizados. Cuestiones como la incorporación de contenidos y la comunicación con docentes y compañeras/os empeoraron por las transformaciones vinculadas a los procesos de virtualización. Esta valoración negativa contrasta con la percepción mayoritariamente positiva del acompañamiento recibido por parte del establecimiento educativo, docentes,

${ }^{21}$ La brecha digital incluye desigualdades entre los estudiantes, tanto de competencias específicas de aprovechamiento de tecnologías de la información y la comunicación (TICs), así como también diferencias de acceso a equipamiento (CEPAL, 2020). 
pares y miembros del hogar. Esto ilustra un estado de situación en el que conviven, y se superponen: a) una especial valoración del acompañamiento recibido durante la suspensión de las clases presenciales; b) el reconocimiento de un avance significativo del uso de las TICs en los procesos educativos; y c) la persistencia de dificultades respecto a ciertos aprendizajes esperados según el nivel educativo.

\section{CUADRO 3. VALORACIÓN DEL ACOMPAÑAMIENTO RECIBIDO Y VALORACIÓN DE} ASPECTOS EDUCATIVOS PRE Y DURANTE LA PANDEMIA

\begin{tabular}{|l|c|c|c|c|c|}
\hline $\begin{array}{c}\text { Valoración pre y } \\
\text { durante la } \\
\text { pandemia }\end{array}$ & Contenidos & $\begin{array}{c}\text { Relación con } \\
\text { docentes y } \\
\text { compañeras/os }\end{array}$ & $\begin{array}{c}\text { Org. del } \\
\text { tiempo para } \\
\text { realizar } \\
\text { tareas }\end{array}$ & $\begin{array}{c}\text { Plataformas } \\
\text { educativas }\end{array}$ & $\begin{array}{c}\text { Reuniones } \\
\text { virtuales }\end{array}$ \\
\hline Peor que antes & $44 \%$ & $33 \%$ & $39 \%$ & $5 \%$ & $4 \%$ \\
\hline Igual que antes & $41 \%$ & $41 \%$ & $35,5 \%$ & $25 \%$ & $18 \%$ \\
\hline Mejor que antes & $10 \%$ & $21 \%$ & $22 \%$ & $55 \%$ & $59 \%$ \\
\hline Comencé a hacerlo & $5 \%$ & $5 \%$ & $3,5 \%$ & $15 \%$ & $19 \%$ \\
\hline Total & $100 \%$ & $100 \%$ & $100 \%$ & $100 \%$ & $100 \%$ \\
\hline
\end{tabular}

Fuente: Elaboración propia, sobre la base de resultados de la encuesta.

Queda en evidencia que la pandemia ha causado cambios en los procesos educativos de las/os jóvenes. Entre las desigualdades que afectan estos procesos y se agravan a partir del Covid-19, se destacan los bajos niveles de acceso a internet y a dispositivos digitales, pues persisten brechas considerables en el acceso efectivo al mundo digital (OIT, 2020; CEPAL, 2020). A continuación, en el último apartado de esta sección se abordará el aspecto de la conectividad a internet de las/los jóvenes encuestadas/os.

En 2020 la provincia contaba con un acceso a internet fijo del 76\% de los hogares, que es una alta proporción de cobertura comparado con el resto del 
país $^{22}$. Pero no es suficiente tener acceso a internet, porque las modalidades de estudio dependen en gran medida de la calidad de la conexión y el tipo de dispositivo (Trucco y Palma, 2020). La velocidad de bajada de datos de internet es uno de los elementos que incide en la calidad de la conexión al ciberespacio. En Tierra del Fuego, la velocidad promedio de bajada de datos de internet fijo en el cuarto trimestre de 2020 fue de 8 megabytes por segundo (mbps), que si se compara con los 14 mbps promedio de velocidad de bajada de todas las provincias del país resulta significativamente inferior ${ }^{23}$. Durante la pandemia, esta situación se tradujo en dificultades y obstáculos para que las/os jóvenes lleven adelante sus actividades cotidianas: nuestra encuesta arroja que el $43 \%$ de las/os jóvenes afirmaron que la principal dificultad para llevar adelante sus actividades en la pandemia fue la falta de conectividad, que coincide con la principal problemática percibida por docentes y alumnas/os de la ENPCP (2020).

A pesar de la defectuosa calidad de la conexión a internet, la encuesta muestra, como se mencionó, que un $86 \%$ de las/os jóvenes están digitalmente conectados, pues poseen $W i-F i$ y PC, notebook o netbook; mientras que un $11 \%$ posee paquete de datos móviles y celular o tablet. Estos datos concuerdan con el $76 \%$ de hogares con acceso a internet fijo en la provincia (ENACOM, 2020), mencionado anteriormente, y con el 71\% de hogares de la Patagonia con acceso fijo a internet $-45 \%$ con buena calidad de señal y $28 \%$ con problemas de señal(ENPCP, 2020). Resulta evidente que la provincia se encuentra dentro de las regiones con mejores niveles de cobertura de internet del país, si bien no sucede lo mismo con la calidad de la conexión.

${ }^{22}$ Según datos del Ente Nacional de Comunicaciones (ENACOM, 2020), la región centro del país tiene una penetración de internet fijo que ronda entre los 65 y 75 hogares de cada 100. El noroeste ronda en los 50 hogares de cada 100. El litoral ronda poco más de 40 hogares de cada 100 .

${ }^{23}$ Elaboración propia sobre la base de Datos Abiertos del ENACOM. 


\subsection{SALUD}

En esta sección se describen: a) las alteraciones sucedidas en la realización de actividad física, los hábitos de sueño y descanso, los modos de alimentación y de consumo de bebidas alcohólicas; b) las sensaciones experimentadas.

Las medidas de restricción a la circulación pueden haber favorecido la conducta sedentaria o reducido la actividad física regular con su consecuente incidencia en la salud. Al consultar por este efecto, un cuarto respondió que el aislamiento no influyó (26\%) y un 35\% que incidió positivamente, ya que durante la pandemia comenzó a realizarla o incrementó la frecuencia con que la hacía previo al brote de Covid-19. Podríamos pensar que el aislamiento, en algunos casos, permitió disponer de mayor tiempo para realizar actividad física. Asimismo, durante el período de mayores restricciones se observó la circulación de rutinas de acondicionamiento físico en redes sociales o programas de televisión que pueden haber estimulado positivamente su realización. Finalmente, un 39\% manifestó que el aislamiento empeoró su vínculo con la actividad porque debieron abandonar su entrenamiento o desarrollarlo en menor medida. Esto pudo incidir negativamente en la salud física y mental.

Respecto a los hábitos de sueño, la mayoría indicó alteraciones, tanto en la cantidad de horas como en la calidad de descanso respecto a la situación previa al inicio de la pandemia. Un 41\% manifestó que duerme más horas que antes, un $30 \%$ menos y un 29\% la misma cantidad. La calidad de descanso empeoró para casi la mitad de las/os respondientes (49\%); sin embargo, para poco más de un tercio no hubo cambios $(35 \%)$ y solo un $16 \%$ manifestó que mejoró. De aquellas/os que consideraron que su calidad de descanso empeoró, un 49\% manifestó que también lo hizo su vínculo con la realización de actividad física; y de quienes manifestaron que su calidad de descanso mejoró, un 52\% señaló que también mejoró su vínculo con la actividad física. A modo de hipótesis, puede 
sostenerse que, si bien los cambios de vida habilitaron mayor tiempo, la prevalencia de sentimientos negativos, el estado de preocupación e incertidumbre atravesado y la drástica alteración de la cotidianeidad tuvieron un impacto negativo en el descanso para una porción importante.

En relación con los efectos en la alimentación, vale mencionar la importante variación de los precios de los alimentos y bebidas no alcohólicas. En la región patagónica (en la que se encuentra Tierra del Fuego), en diciembre de 2020 alcanzó el 36\% con respecto al mismo mes de 2019 (INDEC, 2020), siendo los alimentos frescos aquellos que experimentaron un mayor aumento, particularmente verduras, frutas y carnes. Cabe destacar que el 99\% de las frutas y verduras consumidas en la provincia proviene de otras regiones del país, debiendo atravesar más de $2.000 \mathrm{~km}$ y cuatro aduanas para ingresar a la isla (Livraghi et al., 2021). Las restricciones establecidas a la circulación que dificultaron su llegada, sumado a los incrementos en sus precios, incidieron negativamente en la alimentación de un sector de la población. De este modo, más de un tercio de las/os encuestados manifestó que empeoró su alimentación (39\%), un $44 \%$ que se mantuvo igual y un 17\% que mejoró. Seguramente, la disminución de los ingresos afectó la compra de ciertos alimentos (probablemente de alimentos frescos, ya que fueron los que más se encarecieron) y el aumento de productos no perecederos (que habitualmente están por debajo de las recomendaciones de las guías alimentarias). Precisamente, de quienes indicaron que su alimentación empeoró, un tercio señaló una merma en sus ingresos $(33 \%)^{24}$.

Con el propósito de identificar si hubo cambios en el consumo de bebidas alcohólicas, se consultó por la frecuencia pre y en pandemia. Un 43\% no vio

\footnotetext{
${ }^{24}$ A partir de estos datos, puede suponerse que el empeoramiento en los hábitos alimentarios de las/os jóvenes de sectores económicamente bajos es significativamente mayor.
} 
alterado su consumo, mientras que otro $43 \%$ indicó que disminuyó y solo un 14\% que aumentó. Es posible plantear que tal disminución se asocia a las dificultades para desarrollar prácticas sociales de consumo debido a las restricciones: por un lado, el cierre de bares y discotecas bailables, por el otro, la imposibilidad de realizar reuniones sociales en viviendas. Si bien no se registraron diferencias significativas entre varones y mujeres en cuanto a las variaciones en el volumen de alcohol consumido, identificamos una merma levemente mayor por parte de las mujeres $(46 \%)$ frente a los varones $(38 \%)$.

Con relación a los sentimientos experimentados, observamos una prevalencia de sensaciones negativas. La tristeza (72\%), la intranquilidad (65\%), el aburrimiento (65\%) y la ansiedad (53\%) son los que acumularon mayor frecuencia de respuesta sin registrar diferencias significativas entre mujeres y varones. Consideramos que la particular configuración sociodemográfica de Tierra del Fuego es un aspecto que se relaciona con los dos primeros sentimientos. Tal como señalan Colombari et al. (2020), al ser la mayor parte de la población joven nacida en otra provincia (64\%), gran parte de las redes familiares se encuentran fuera del territorio insular. La lejanía con los principales centros urbanos del país y las dificultades para el cruce fronterizo argentino/chileno, que impidieron la conectividad terrestre, pueden haber afectado negativamente a las/os jóvenes. Entre otros factores, la tristeza e intranquilidad registrada pudo deberse a la imposibilidad de acercarse a sus familias frente a alguna eventualidad producto de la pandemia.

Con respecto a las preocupaciones, la que mayor frecuencia reúne es la posibilidad de ser diagnosticada/o de Covid-19 positivo o que un ser querido reciba dicho diagnóstico (56\%). La segunda más señalada es la falta de ingresos $(50 \%)$. Del grupo que indicó dicha preocupación, la mayor parte experimentó una reducción (54\%). Al observar los sentimientos experimentados por dicho grupo, no sorprende que casi tres cuartas partes se sintió intranquilo (71\%) y más de la mitad, ansioso (55\%). Estos datos permiten observar que aquellas/os 
jóvenes que experimentaron una reducción de sus ingresos son quienes también atravesaron una mayor situación de padecimiento psíquico debido a la experimentación de sentimientos de intranquilidad y ansiedad producto de su preocupación por la economía doméstica.

CUADRO 4. EFECTOS DE LA PANDEMIA Y EL AISLAMIENTO EN LA SALUD DE LAS/OS JÓVENES RESPONDIENTES

\begin{tabular}{|c|c|c|c|c|}
\hline \multicolumn{2}{|c|}{$\begin{array}{c}\text { Incidencia del aislamiento en la realización de } \\
\text { actividad física }\end{array}$} & \multicolumn{3}{|c|}{$\begin{array}{c}\text { Alteración en los hábitos } \\
\text { alimentarios }\end{array}$} \\
\hline Incidió positivamente & $35 \%$ & $\begin{array}{l}\text { Mejoró sus hábitos } \\
\text { alimentarios }\end{array}$ & & $\begin{array}{l}17 \\
\%\end{array}$ \\
\hline Incidió negativamente & $39 \%$ & $\begin{array}{l}\text { Empeoró sus hábitos } \\
\text { alimentarios }\end{array}$ & & $\begin{array}{l}39 \\
\%\end{array}$ \\
\hline No incidió & $26 \%$ & No experimentó cam & & $\begin{array}{l}44 \\
\%\end{array}$ \\
\hline Total & $100 \%$ & & otal & $\begin{array}{c}100 \\
\%\end{array}$ \\
\hline \multicolumn{2}{|l|}{ Alteración del descanso } & $\begin{array}{c}\text { Sentimientos } \\
\text { experimentados }\end{array}$ & Sí & No \\
\hline Mejoró la calidad de descanso & $16 \%$ & Tristeza & $\begin{array}{l}72 \\
\%\end{array}$ & $\begin{array}{r}28 \\
\%\end{array}$ \\
\hline Empeoró la calidad de descanso & $49 \%$ & Intranquilidad & $\begin{array}{l}65 \\
\%\end{array}$ & $\begin{array}{l}35 \\
\% \\
\end{array}$ \\
\hline No experimentó cambios & $35 \%$ & Aburrimiento & $\begin{array}{l}65 \\
\% \\
\end{array}$ & $\begin{array}{l}35 \\
\% \\
\end{array}$ \\
\hline Total & $100 \%$ & Ansiedad & $\begin{array}{l}53 \\
\%\end{array}$ & $\begin{array}{l}47 \\
\%\end{array}$ \\
\hline
\end{tabular}

Fuente: Elaboración propia, sobre la base de resultados de la encuesta.

\subsection{OCIO Y USO DEL TIEMPO LIBRE}

La última de las dimensiones indagadas en la encuesta fueron las actividades de ocio y el uso del tiempo libre durante la pandemia, un aspecto de la vida cotidiana que también se vio muy afectado por el drástico cambio de 
rutinas asociado al confinamiento en la vivienda. El cuestionario se orientó a relevar a qué actividades le dedicaron más o menos tiempo las/os jóvenes y cuáles comenzaron a realizarse o dejaron de realizarse en relación con el contexto previo.

Para presentar los hallazgos de nuestro estudio, en primer lugar, cabe reconocer que desde hace unos años nuestras prácticas de ocio y entretenimiento están cada vez más mediadas por los entornos digitales, y por una interacción entre medios digitales, tradicionales y actividades no virtualizadas, en virtud de la masificación en el acceso a ciertas tecnologías vinculadas con la digitalización, principalmente dispositivos y conectividad móvil. En Argentina, los datos disponibles sobre consumos culturales en el contexto prepandemia indican que casi la totalidad de las/os jóvenes tiene celular (96,2\%) y usa internet diariamente (95,9\%) (SINCA, 2017).

En ese marco, no resulta llamativo que el consumo de contenidos audiovisuales vía streaming (96\%) y el uso de redes sociales $(96,5 \%)$ sean actividades realizadas prácticamente por la totalidad de las/os encuestados: se trata de prácticas de ocio que se han extendido entre toda la población, y especialmente entre las/os jóvenes. En relación con ello, durante el período de aislamiento, en el que muchas otras prácticas de la vida cotidiana se vieron imposibilitadas por las restricciones de circulación, tales actividades han estado entre las más elegidas, ya sea porque aumentó el volumen de tiempo dedicado a ellas, o bien porque comenzaron a realizarse: $52 \%$ consume más contenido audiovisual vía streaming que antes o comenzó a consumirlo, y 50,5\% usa más las redes sociales o comenzó a usarlas.

Sin embargo, nuestro estudio indica que otro tipo de actividades de ocio y uso del tiempo libre también tuvieron gran incidencia durante la pandemia: un $58 \%$ indicó que prueba nuevas recetas con mayor frecuencia o que no lo hacía antes y comenzó a hacerlo (es la actividad más mencionada entre las que se hacen 
más o comenzaron a realizarse); y 34\% manifestó que practica deportes al interior de su vivienda con más frecuencia o que comenzó a hacerlo. En ese sentido, a pesar de lo que plantean algunas posturas adultocéntricas, las actividades vinculadas con el denominado «ocio pasivo»y con el «ocio digital» han concentrado el interés de las/os jóvenes en su tiempo libre, pero no lo han monopolizado durante el período de aislamiento ${ }^{25}$.

Por otro lado, es en las actividades que dejaron de hacerse o se realizan en menor medida donde se constata un efecto aún más decisivo del confinamiento en la vida cotidiana de las/os jóvenes, en tanto la posibilidad de elegir tales actividades en función de su propio deseo e interés se ha visto seriamente limitado. Es el caso, principalmente, de las reuniones sociales, a las que un 67,5\% de las/os respondientes asiste menos que antes o ha dejado de asistir; y de los deportes al exterior, que un $44 \%$ practica en menor medida o ha dejado de practicar.

${ }^{25}$ Conceptualmente, se denomina ocio pasivo a aquellas actividades en las cuales resulta necesario precisar el nivel de participación que estas suponen, entendiendo por «participación» algún tipo de involucramiento corporal o mental en el desarrollo de la actividad (Cuenca Cabeza, 2000). Usualmente, refiere a un tipo de ocio que se opone al denominado «ocio activo», vinculado a la realización de actividades como deportes, prácticas artísticas, etc. Si bien se trata de un concepto discutido, la idea tiene vigencia en el sentido común, y su connotación negativa ha tendido a profundizarse en relación con las miradas tecnofóbicas sobre el ocio digital, o e-ocio, es decir, aquel que se apoya centralmente en el uso de herramientas tecnológicas y entornos digitales (Viñals, Abad y Aguilar, 2014). 


\section{CUADRO 5. ACTIVIDADES DE OCIO DESARROLLADAS DURANTE EL}

AISLAMIENTO

\begin{tabular}{|l|r|r|r|r|}
\hline $\begin{array}{c}\text { Actividades } \\
\text { desarrolladas } \\
\text { durante el } \\
\text { aislamiento }\end{array}$ & $\begin{array}{c}\text { Se hace más } \\
\text { o comenzó a } \\
\text { hacerse }\end{array}$ & $\begin{array}{c}\text { Se hace igual } \\
\text { que antes }\end{array}$ & $\begin{array}{c}\text { Se hace } \\
\text { menos o dejó } \\
\text { de hacerse }\end{array}$ & \multicolumn{1}{|c|}{ Total } \\
\hline Probar nuevas recetas & $61 \%$ & $27 \%$ & $12 \%$ & $100 \%$ \\
\hline $\begin{array}{l}\text { Consumir contenidos } \\
\text { audiovisuales por } \\
\text { streaming }\end{array}$ & $52 \%$ & $41 \%$ & $7 \%$ & $100 \%$ \\
\hline Usar redes sociales & $51 \%$ & $42 \%$ & $8 \%$ & $100 \%$ \\
\hline $\begin{array}{l}\text { Practicar deportes al } \\
\text { interior }\end{array}$ & $39 \%$ & $23 \%$ & $38 \%$ & $100 \%$ \\
\hline $\begin{array}{l}\text { Practicar deportes al } \\
\text { exterior }\end{array}$ & $21 \%$ & $21 \%$ & $58 \%$ & $100 \%$ \\
\hline $\begin{array}{l}\text { Asistir a reuniones } \\
\text { sociales }\end{array}$ & $9 \%$ & $20 \%$ & $71 \%$ & $100 \%$ \\
\hline
\end{tabular}

Fuente: Elaboración propia, sobre la base de resultados de la encuesta.

En ese sentido, podría considerarse que el aumento que se constata en el uso de redes sociales puede estar vinculado a la virtualización del contacto social, principalmente durante las primeras semanas de la pandemia, en las que el aislamiento fue muy estricto. En el caso de Tierra del Fuego, además, la progresiva flexibilización de algunas restricciones - que permitió, por ejemplo, la realización al aire libre de reuniones sociales y deportes- coincidió con el período más intenso del invierno, dificultando así el aprovechamiento de las posibilidades habilitadas. Asimismo, si se analiza el tipo de actividades y el tiempo dedicado a ellas en relación con los rangos etarios, lo que se observa es que fueron las/os más jóvenes de la muestra (18 a 21 años) quienes más transformaron sus prácticas de ocio y uso del tiempo libre, tanto respecto a las actividades que más aumentaron su frecuencia como a las que menos lo hicieron. Podemos pensar que esto da cuenta de la centralidad de la actividad educativa presencial en la organización de la vida cotidiana de este subgrupo, ya que más 
de la mitad de las/os respondientes de esta edad son inactivas/os (54,5\%). A la vez, da cuenta de que la virtualización de la educación y del trabajo no supuso necesariamente la posibilidad de ampliar el tiempo destinado al ocio.

A modo de síntesis, lo que estos datos ponen de relieve es que la pandemia ha tenido un fuerte impacto en el desarrollo de las prácticas de uso del tiempo libre para las/os jóvenes encuestados, pues las restricciones al contacto social, la circulación y el uso de los espacios públicos se suman a otros condicionamientos sociales, económicos y culturales preexistentes que inciden sobre sus posibilidades de desarrollar experiencias subjetivas significativas, elegidas libremente, gozosas y autotélicas (Viñals, Abad y Aguilar, 2014).

\section{CONSIDERACIONES FINALES}

Es altamente probable que la doble crisis en el mercado de trabajo fueguino (por la pandemia y por la recesión económica prepandemia) haya modificado negativamente el rumbo de las trayectorias laborales recorridas con su consecuente impacto sobre las economías domésticas-, afectando las posibilidades de emancipación familiar y su salud psíquica. Diversos datos del trabajo contribuyen a sostener esta hipótesis, ya que, por un lado, una proporción no menor de jóvenes debió mudarse en su ciudad o regresar a la provincia a causa del Covid-19; y por otro, la falta de ingresos fue una de las principales preocupaciones expresadas, especialmente por quienes experimentaron a la vez sentimientos de intranquilidad y ansiedad. Precisamente, atendiendo a la especial vulnerabilidad de la población juvenil producto de esta doble crisis del mercado laboral, el gobierno argentino lanzó en marzo de 2021 un nuevo programa denominado «Potenciar Inclusión Joven», consistente en una asignación mensual 
directa de recursos destinada a personas de 18 a 29 años para financiar proyectos socioproductivos y sociolaborales ${ }^{26}$.

En el plano educativo, la crisis sanitaria imposibilitó el dictado normal de clases, y a pesar de que en muchos casos las actividades educativas se retomaron de manera virtual, las/os encuestados percibieron que sus aprendizajes empeoraron. Teniendo en cuenta los altos niveles de penetración de internet, la valoración positiva vinculada al desarrollo de competencias en el uso de herramientas digitales y el múltiple acompañamiento para el sostenimiento de las actividades educativas, resulta relevante señalar la percepción negativa acerca de los aprendizajes en el contexto de virtualización forzada. Si bien muchas/os jóvenes continuaron sus estudios, se podría sostener que la trayectoria recorrida ha sido muy distinta a la proyectada.

Hemos identificado una serie de efectos producto de la pandemia que afectaron hábitos saludables de vida. Entre estos, señalamos que: a) una proporción importante empeoró su relación con la realización de actividad física, lo que tuvo consecuencias en el desmejoramiento de la calidad de descanso; b) más de un tercio de las/os jóvenes respondientes vio deteriorada su alimentación. Al indagar sobre los sentimientos atravesados durante los primeros meses de la pandemia, la tristeza y la intranquilidad fueron los que registraron mayor frecuencia de respuesta. Las dificultades laborales y educativas inciden de modo negativo en las proyecciones a futuro. Asimismo, las preocupaciones derivadas por la lejanía de las redes familiares en un contexto de crisis agregan mayor pesadumbre. A esta sensación pueden haber contribuido los efectos del confinamiento en las limitaciones y condicionamientos, a la hora de elegir actividades en función de su propio deseo e interés en relación con el uso del tiempo libre.

\footnotetext{
${ }^{26}$ Fuente: https://www.argentina.gob.ar/desarrollosocial/potenciarinclusionjoven
} 
Es evidente que la situación epidemiológica produjo profundas consecuencias en muchos aspectos de la vida de algunas/os jóvenes. Podemos pensar que estos cambios impactaron de lleno en sus proyectos vitales, en los tránsitos emancipatorios en términos familiares, laborales y educativos que atraviesan por su condición juvenil. En este sentido, proponemos, a modo de hipótesis, que la crisis por Covid-19 se constituyó en tanto marca generacional al trastocar proyecciones de vida para un sector importante de la población que atraviesa un momento del ciclo vital caracterizado por los tránsitos mencionados. Más allá de las limitaciones de la indagación, el estudio ofrece un registro multidimensional de los efectos que la pandemia, en tanto suceso sociohistórico de escala global, ha tenido en la vida de las/os jóvenes. Asimismo, permite captar y describir sus consecuencias con relación a algunos aspectos singulares del territorio. De esta manera, contribuye a la formulación de propuestas situadas a partir de diagnósticos de base empírica. Pretendemos que el presente trabajo estimule futuras líneas de indagación en torno a esas trayectorias interrumpidas y los posibles rumbos que estas tomen a futuro.

Con relación al trabajo y los ingresos, resulta fundamental continuar relevando la situación en cuanto a los ingresos de las/os jóvenes de Tierra del Fuego, para valorar los efectos a mediano y largo plazo de la caída de la actividad asociada a la pandemia y el aislamiento durante 2020, en especial en una economía poco diversificada como la local. Desde un punto de vista cualitativo, sería interesante profundizar en la cuestión de las asistencias estatales que alcanzaron a sectores de la población que habitualmente no recurren a ellas. Es de interés describir y comprender cómo fue la experiencia de aquellas/os que se vieron en la necesidad de requerir, tal vez por primera vez, ese tipo de asistencias, y qué posibilidades de sostenimiento y recuperación significaron para las economías familiares, así como el modo en que las asignaciones ofrecidas por el Estado durante la pandemia se articulan y articularán con nuevas políticas públicas — como el programa de inclusión laboral mencionado - para ampliar y 
mejorar las expectativas y proyecciones a futuro de estas/os jóvenes. En este sentido, teniendo en cuenta la crisis económica que la pandemia vino a profundizar, cabría preguntarse acerca de la pertinencia de medidas estatales de carácter estable que contribuyan a la disminución de la vulnerabilidad laboral y de ingresos descrita, puesto que el aspecto transitorio de los programas de emergencia como el IFE no disminuyen los altibajos de las economías familiares.

En lo que refiere a la educación, el contraste entre la valoración positiva de las/os jóvenes — por ejemplo, del acompañamiento recibido para continuar sus estudios - y la valoración negativa de aspectos sustantivos de los procesos de formación — como el aprendizaje de contenidos—, invita a profundizar la indagación en torno a la virtualización. Más información — de carácter cualitativo - acerca de las dificultades experimentadas permitirá capitalizar la experiencia, diseñando estrategias a futuro orientadas a fortalecer la formación de quienes se vieron obligadas/os a transitar parte de su recorrido en un escenario de virtualización forzosa, así como a la incorporación de TICs a la educación (superior, en nuestro caso) que apunten a evitar o superar tales dificultades en los escenarios presenciales/virtuales que planteará la nueva normalidad. En el caso de Tierra del Fuego, las propuestas deben incorporar la situación particular de alto nivel de cobertura de internet con bajo nivel de calidad de la conexión.

RECIBIDO: 18 DE JUNIO DE 2021

ACEPTADO: 28 DE JULIO DE 2021

\section{BIBLIOGRAFÍA}

Bidaseca, K., Aragão Guimarães Costa, M., Brighenti, M. y Ruggero, S. (2020). Diagnóstico de la situación de las mujeres rurales y urbanas, y disidencias en el contexto de COVID-19. (Informe de la Unidad Coronavirus). Ministerio de Ciencia, Tecnología e Innovación, Ministerio de las Mujeres, Géneros y Diversidad, Consejo Nacional de Investigaciones Científicas y Técnicas. 
Bottaro, H., Ejarque, M., Pérez Centeno, M. Preda, G., Livraghi, E. C. E. y SPONTÓn, E. A. (2021). La producción agropecuaria de Tierra del Fuego y sus cambios en el periodo intercensal 2002-2018. Realidad Económica, (339), 155-200.

Colombari, B., Hermida, M. y Guzzi, L. (2020). Gestionar la pandemia: la experiencia del Municipio de Ushuaia. Cuadernos de Investigación. Serie Economía, № 9, 79-98.

Colombari, B., Hermida, M. y Picón, J. (2019). Juventud, ¿divino tesoro? Jóvenes y trabajo en Tierra del Fuego. 2003-2018. Sociedad Fueguina, (6), 6-20.

Colombari, B., Hinca, L., Traba, J. y Andrade, A. (2020). Promover el arraigo: políticas de juventud en Tierra del Fuego 1991-2018. Fuegia, $3(1), 21-36$.

COMISIÓn ECONÓMICA PARA AMÉRICA LATINA Y El CARIBE (2021). Panorama Social de América Latina 2020. Santiago: autor.

COMISIÓN ECONÓMICA PARA AMÉRICA LATINA Y EL CARIBE/ORGANIZACIÓN DE LAS NACIONES UNIDAS PARA LA EDUCACIÓN, LA CIENCIA Y LA CULTURA (2020). La educación en tiempos de la pandemia de COVID 19 (Informe COVID-19 CEPAL-UNESCO). Santiago: autor.

Cuenca Cabeza, M. (2000). Ocio humanista. Dimensiones y manifestaciones actuales del ocio. Bilbao: Universidad de Deusto.

Ente Nacional de Comunicaciones (2021). Datos Abiertos-Acceso a internet.

Guzzi, L., Colombari, B., Picón, J. y Hermida, M. (2020). \#ushuaiafindelmundo. Turismo, pandemia y Estado. Sociedad Fueguina, (7), 13-23.

Instituto Nacional de Estadísticas y Censos (2010). Censo Nacional de Población, Hogares y Viviendas.

. (2019). Mercado de Trabajo. Tasas e indicadores económicos (EPH) (Informes técnicos, Vol. 3, No 232). Buenos Aires: autor.

. (2020). Índices de precios al Consumidor (IPC) (Informes técnicos, Vol. 5, No 7). Buenos Aires: autor.

JACINTO, C. (2010). La construcción social de las trayectorias laborales de jóvenes. Buenos Aires: Teseo. 
KRAUSKOPF, D. (2004). Perspectivas sobre la condición juvenil y su inclusión en las políticas públicas. En E. Gerber y S. BALARdini (eds.), Políticas de juventud en Latinoamérica. Argentina en perspectiva. FLACSO.

Malegarie, J. y Fernández, P. E. (agosto, 2019). Técnicas y tecnologías: encuestas vía web, desafíos metodológicos en el diseño, campo y análisis. En Mesa Metodología de la investigación sociológica y epistemología. XIII Jornadas de Sociología. Facultad de Ciencias Sociales, Universidad de Buenos Aires, Buenos Aires, Argentina.

Martín Criado, E. (2009). Juventud. En Diccionario Crítico de Ciencias Sociales (pp. 1630-1635). Madrid: Universidad Complutense de Madrid.

Ministerio De DeSARRollo Social (2021). Potenciar Inclusión Joven.

Ministerio De EdUCACIÓN (2020). Evaluación Nacional del Proceso de Continuidad Pedagógica (Informe Preliminar Encuesta a Hogares). Buenos Aires: Secretaría de Evaluación e Información Educativa/UNICEF.

ORgANIZACIÓN INTERNACIONAL DEL TRABAJO (2020). Los jóvenes y la Covid19: efectos en los empleos, la educación, los derechos y el bienestar mental (Informe de la encuesta 2020).

RODRÍGUEZ ENRÍQUEZ, C. (2007). Economía del cuidado, equidad de género y nuevo orden económico internacional. En Del Sur hacia el Norte: Economía política del orden económico internacional emergente (pp. 229-240). Buenos Aires: CLACSO.

Sistema de Información Cultural de la Argentina (SINCA) (2017). Los jóvenes y los consumos culturales (Informe de inv. Sobre la base de la Encuesta Nacional de Consumos Culturales 2017).

Trucco, D. y Palma, A. (2020). Infancia y adolescencia en la era digital: un informe comparativo de los estudios de Kids Online del Brasil, Chile, Costa Rica y el Uruguay (Documentos de Proyectos, Estudios e Investigaciones). Santiago: CEPAL.

Viñals Blanco, A., Abad Galzacorta, M. y Aguilar Gutiérrez, E. (2014). Jóvenes conectados. Una aproximación al ocio digital de los jóvenes españoles. Communication Papers, (4), 52-68.

Vommaro, P. (2014). Juventudes, políticas y generaciones en América Latina: acercamientos teórico conceptuales para su abordaje. En P. VOMMARO y S. Alvarado (eds.), En busca de las condiciones juveniles latinoamericanas. CLACSO. 